\title{
The Atlas Liquid Argon Calorimeter at the CERN Large Hadron Collider: General Performance and Latest Developments of the High Voltage System
}

\author{
Valerio Grassi, Senior Member, IEEE, on behalf of the ATLAS LAr Calorimeter group
}

Abstract-The ATLAS experiment is designed to study the proton-proton collisions produced at the Large Hadron Collider (LHC) at CERN. Liquid argon sampling calorimeters are used for the identification and characteristics measurement of the electrons, photons and hadronic jets. Since the first collisions in 2010 , the calorimeters operated almost optimally, playing a leading role in the discovery of the Higgs boson announced in July 2012. A special emphasis in this paper will be given on the latest developments of the high voltage power supply system. These efforts allowed to equip the calorimeters with innovative power supply modules able to temporarily sustain high current load without major voltage drop.

\section{INTRODUCTION}

HE ATLAS experiment [1] is a multipurpose particle physics detector at the LHC proton-proton collider at CERN. The detector has a wide physics program, spanning from precision measurements of $\mathrm{W} \pm$ bosons, top and bottom quarks properties, to searches for the Higgs boson and supersymmetric particles.

\section{THE LAR CALORIMETER SYSTEM}

The Liquid Argon (LAr) calorimeter system plays a key role in measuring energy, position and time of electrons, photons and charged hadrons.

The LAr calorimeters [2],[3] are housed in three cryostats, one barrel and two end-caps (Fig. 1). The barrel cryostat contains the electromagnetic (EM) barrel calorimeter that covers the pseudorapidity range $0<|\eta|<1.4$. It is a leadliquid argon sampling calorimeter with readout electrodes built in an accordion shape in order to provide a full coverage in $\varphi$. It is divided into three longitudinal layers called (starting from smaller radii): strip, middle and back layers. In front of the calorimeter, a pre-sampler made of a $1 \mathrm{~cm}$ layer of liquid argon is used to estimate the energy loss due to the material upstream of the calorimeter.

Each endcap cryostat includes three detectors: the electromagnetic endcap (EMEC), the hadronic endcap (HEC) and the forward calorimeter (FCAL). The EMEC

Valerio Grassi is with the State University of New York at Stony Brook, NY, USA (e-mail: valerio.grassi@cern.ch).
42 utilizes the same technique as in the EMB, with the 43 electrodes arranged in a wheel-like geometry. It is 44 subdivided into outer and inner wheels, covering the 45 regions $1.375<|\eta|<2.5$ and $2.5<|\eta|<3.2$, respectively. 46 A presampler is placed in front of the EMEC in the region $47 \quad 1.5<|\eta|<1.8$.

48 The hadronic endcap covers the pseudorapidity range of 1.5 $49<|\eta|<3.2$. It is divided into four longitudinal layers with 50 planar geometry and uses copper as absorber. The forward 51 calorimeter covers the high pseudorapidity regions: $3.1<|\eta|$ $52<4$.9. It consists of three layers: one electromagnetic layer 53 with copper as passive material and two hadronic layers 54 using tungsten as absorber material.

55

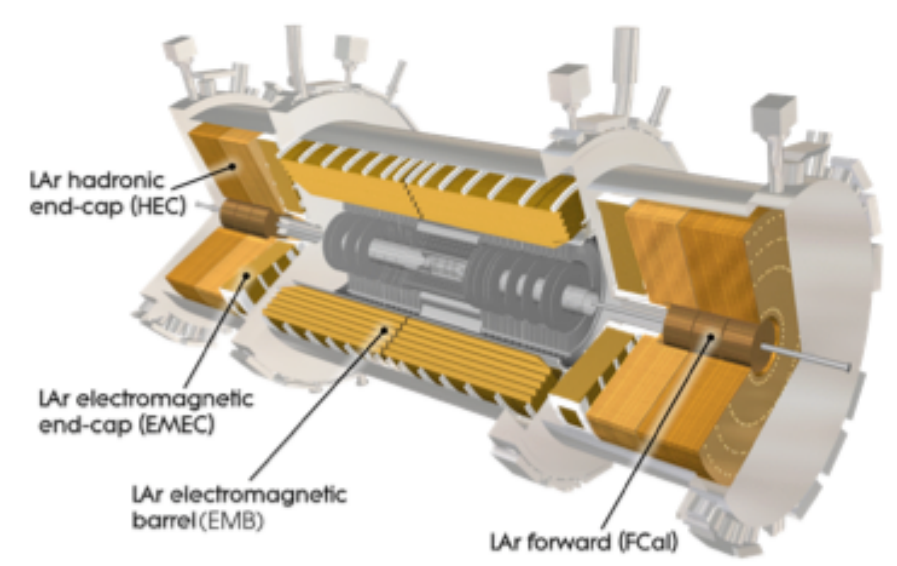

Fig. 1. The Atlas liquid Argon Calorimeter system.

\section{PRINCIPLE OF OPERATION AND SIGNAL READOUT}

The ionization signal is produced by the charged particles crossing the liquid argon gap in the calorimeters while drifting through a high electric field generated by the HighVoltage (HV) system between the absorber plates and readout electrodes, Fig.2 . This produces a triangle shaped ionization pulse shown in Fig.3. This signal is then processed by front-end boards where it is transmitted through two paths, an analog one is used for the Level 1 calorimeter trigger system, and a digital one which is used for physics reconstruction. In both paths the signal is shaped with a bipolar filter CR-RC ${ }^{2}$ to optimize the signal- 
to-noise ratio [1]. In the digital path the signal is splitted into three overlapping linear gain scales, in order to cope with the large dynamic range required for the expected physics signals. At this level, it is temporarily stored in an analog pipeline (Switched Capacitor Array, SCA). Finally, if the signal is accepted by the Level 1 trigger, the analog value read from the SCA is digitized by means of a 12-bit $\mathrm{ADC}$ and transferred with the proper gain to the Back-End electronics in the ATLAS technical cavern, where the energy and timing are reconstructed.

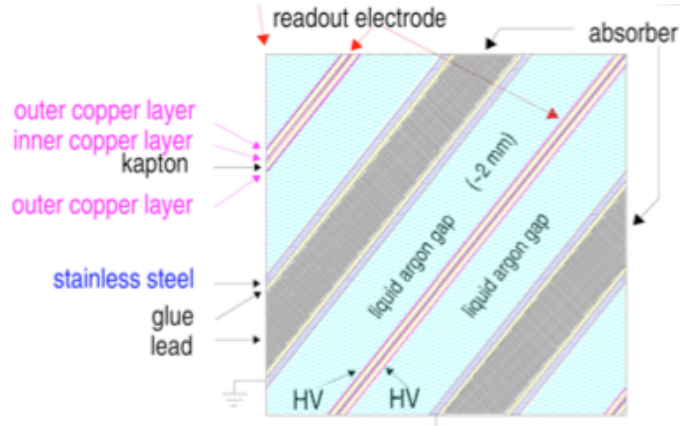

Fig. 2. Structure of the electrodes of the LAr calorimeter (accordion shape)

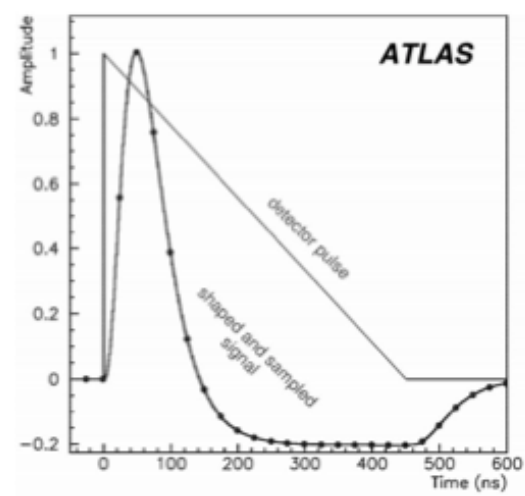

Fig. 3. Detector pulse, shaping and digitization

Calibration boards, installed in the front-end crates, provide the calibration of the whole electronic chain by means of the injection of a well known exponential pulse into the system.

\section{THE LAR HV VOLTAGE SYSTEM}

The LAr High-Voltage system is designed to create an electric field $\mathrm{E}$ in the calorimeter liquid argon gaps that allows the drift of ionization charge induced by the interaction of charged particles while traversing the calorimeter. The choice of the operational regime of the HV system is motivated by two main reasons: the drift time of the ionization electrons and the breakdown voltage of the liquid argon. As the drift velocity of the electrons in the liquid argon gap is approximately proportional to $\mathbf{E}^{1 / 3}$, a weak electric field will increase the drift time of the electrons, increasing the pile-up effect of the shaped signals. If the electric field is too intense, the risk of sparks in the gaps rises, disturbing the operation of the calorimeter with a potential risk for the gap integrity. These considerations have led to the setting of an operational field of approximately $1 \mathrm{kV} / \mathrm{mm}$ across all the calorimeters. In the EMEC the gap size varies with $\eta$ : different voltages are applied in different $\eta$ intervals in order to keep the electric field approximately uniform.

\section{The High Voltage POWER SUPPLIES}

The High Voltage power supplies for the LAr calorimetry are produced by Iseg GmbH (Rossendorf, Germany).

The system is composed by 23 crates, containing 170 modules, feeding 4456 independent HV lines. Each module contains two printed circuit boards with a maximum of 32 independent high voltage channels.

Typically the voltage resolution setting is in the $\mathrm{mV}$ range (from $10 \mathrm{mV}$ to $50 \mathrm{mV}$, depending on the type of module) and the measurement resolution is comparable. The current measurement resolution is a function of the maximum output current per single channel and covers the range 4$200 \mathrm{nA}$. The accuracy in the voltage measurement is guaranteed for one year after calibration as $\pm\left(0.01 \% \cdot V_{\text {out }}\right.$ $+0.02 \% \cdot \mathrm{V}_{\max }$ ) where $\mathrm{V}_{\max }$ is the maximum output voltage of the module. The accuracy in the current measurement is $\pm\left(0.02 \% \cdot I_{\text {out }}+0.02 \% \cdot I_{\max }\right)$ where $I_{\max }$ is the maximum output current of the module. The sampling rate of voltages and currents is typically set at 500 samples/s, and a programmable digital low pass filter is computed over a selectable number of samples $(1,64,128,256,512,1024)$. The voltage ramp up and ramp down (in $\mathrm{V} / \mathrm{s}$ ) is programmable via software in the range from $10^{-6} \times \mathrm{V}_{\max }$ to $0.2 \times \mathrm{V}_{\max }$. The HV modules installed in the system cover the voltage range from $500 \mathrm{~V}$ to $2500 \mathrm{~V}$, with a maximum output current in the range from $75 \mu \mathrm{A}$ (Barrel presampler) to $10 \mathrm{~mA}$ (Forward calorimeters). Most of the units are equipped with a single one-step Greinacher switched resonance converter (switching frequency: $80 \mathrm{kHz}$ ) that feeds sixteen optocoupled series voltage regulators (one per channel), adjustable independently via software (distributor module).

Each module is equipped with a CANbus (Controller Area Network bus) interface, that allows a bi-directional communications for the control of the unit, the firmware upgrade and the upload of the calibration parameters. Moreover, each HV module, acting as a double node (one per board) of a CANbus, is inserted in a crate that contains eight of them. The crate controller unit provides the $24 \mathrm{~V}$ and $5 \mathrm{~V}$ supplies and the routing of the CAN signals to the backplane. The communication between the HV modules and the control PCs is performed by means of an OPC (OLE for process control) client/server approach. The integration with the ATLAS Detector Control System 


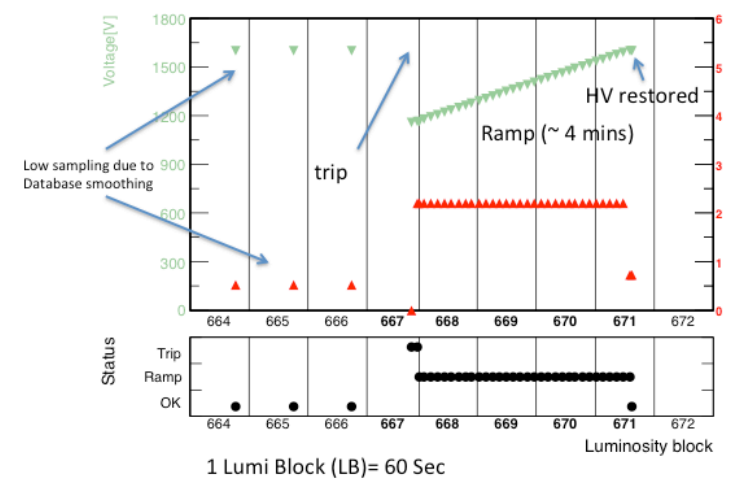

(DCS) is developed by the commercial software package PVSS (now rebranded as Siemens WinCC OA ${ }^{\mathrm{TM}}$ ).

Fig. 4. Response to a trip of an Iseg distributor module in the Hadronic End Cap calorimeter (HEC). After the trip the ramp is set to $2 \mathrm{~V} / \mathrm{sec}$. During the trip time the signal from the region fed by this HV line is not processed

During the LHC operations with collisions, the particles flowing across the calorimeter system can create a massive ionization concentrated in one electrode. This amount of extra charge required by the detector can exceed the maximum current output of one channel of the HV module. This is named Trip.

In the Fig 4. is shown the response to a trip of an Iseg distributor module in the Hadronic End Cap calorimeter. After the trip the ramp is set to $2 \mathrm{~V} / \mathrm{sec}$. This involves a loss of data; in fact during the trip time the signal from the region fed by this $\mathrm{HV}$ line is not processed since it is impossible to accurately assess the corresponding correction factor. On the contrary, the signal taken during the ramp up phase of the HV after a trip is recoverable for physics measurements since a proper correction factor can be applied in this case.

Recently, Iseg has also developed modules equipped with one miniaturized high voltage resonance converter per channel (independent generators). In our system we have implemented two different types of them (16 channels, $3 \mathrm{kV}, 3 \mathrm{~mA}$ each and 32 channels, $3 \mathrm{kV} 1.3 \mathrm{~mA}$ each). The independent generators design allows the power supply to switch each channel from the voltage source mode into a Current-Control mode (CC). This allows to cope the request of a high charge injection in the detector during a massive LAr ionization concentrated in one electrode, without driving the channel in a trip state (with the consequent slow ramping up to the previous set voltage, Fig. 5). In fact, an independent generators module is able to adjust the voltage for a channel in a millisecond range, while delivering the maximum output current allowed by the internal HV power supply. If a channel is permanently shorted, it is possible to set a trip delay action, which turns the channel OFF after a programmed set time.

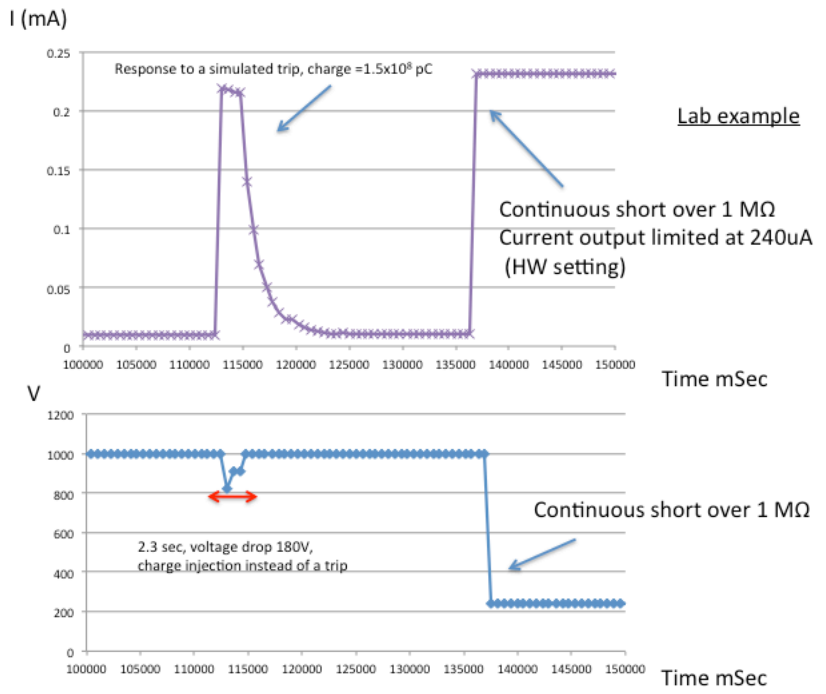

Fig. 5. Test at CERN of the response of a independent generator module to a simulated trip due to an injected charge of $1.5 \times 10^{8} \mathrm{pC}$. The maximum output current was set to $240 \mu \mathrm{Amps}$. When the channel was continuously shorted to ground over $1 \mathrm{M} \Omega$ resistor, the max output current is delivered and the voltage is adjusted accordingly (Current-Control mode).

\section{LUMINOSITY MONITORING USING THE HV SYSTEM}

During proton-proton collisions at the LHC, a large flux of minimum bias particles traverses the detector forward regions. Minimum bias events are originated from soft $p p$ interactions and consist of mostly $\pi^{0} / \pi^{ \pm}$mesons with low transverse momentum. Most of their energy is deposited in the electromagnetic forward calorimeter as electromagnetic showers of photon pairs from $\pi^{0}$ decays. The induced particle flux intensity is directly proportional to the interaction rate and then to the luminosity. The current injected by the high-voltage system that feeds the electromagnetic layer of the FCAL, in order to compensate the ionization losses from minimum bias events is therefore correlated to the flux, giving a direct handle on the luminosity [4],[5]. This approach has two advantages. The response is linear with the luminosity and it is totally independent from the ATLAS trigger and data acquisition (DAQ) system, in fact it is not affected by event selection biases and DAQ downtime.

This method was found to be reliable and accurate, giving results in optimum agreement with the official ATLAS luminosity monitoring methods (LUCID and the Van der Meer scans) [6].

\section{Performance of the LAR CAlorimeter DURING THE LHC RUN I}

The data quality for the LAr calorimeters has been in constant improvement since the early periods of data taking; more than $99 \%$ of the data taken during the 2012 
proton-proton campaign are suitable for analysis (to be compared with approximately $90 \%$ in 2010 and approximately $97 \%$ in 2011 ), with the main sources of data loss being HV trips $(0.46 \%)$ and noise bursts $(0.2 \%)$. The data rejection due to HV trips is more than halved in the 2012 in comparison to the previous year, as shown in Table1.

As already discussed, if a HV line trips, some data are lost since the HV values are varying too quickly to be properly managed offline. To reduce the impact of these trips on data taking several steps have been taken, such as reducing the $\mathrm{HV}$ on problematic lines and installing the independent generators modules to feed the regions of the inner wheels in both EMECs, where most of the trips occur. In Fig.6 is shown the evolution of the luminosity losses associated to HV trips during 2012 , from $1.8 \%$ to $0.1 \%$. In April 2012 (when the integrated luminosity delivered by the LHC was $0.9 \mathrm{fb}^{-1}$ ) the new current control modules were installed and an immediate reduction of the lost luminosity is seen. The trend remains constant until September 2012 (when the integrated luminosity delivered by the LHC was $14.5 \mathrm{fb}^{-1}$ ) when a hardware problem on a distributor module feeding a large region of the EMEC presampler on the $\mathrm{C}$ side (more than 6 trips in a fill) led to an increase of the lost luminosity around $1 \%$. Once the module has been replaced the trip rate returned to the previous value.

We have also observed an increase of the trip rate after the winter shutdowns, the technical stops or while the LHC has increased significantly the instantaneous luminosity (i.e. increasing the number of proton bunches colliding in ATLAS). After a few fills, the trip rate decreases at the previous level. The origin of this "training" effect due to the collisions is still unclear, one possible explanation could be related to a charge-trapping phenomenon in the insulating kapton layer of the electrodes.

A noise bursts occur when many cells in a region of the calorimeter give large signals with distorted waveforms for a very brief period of time (mostly below $5 \mu \mathrm{s}$ ). The source of the noise is not yet fully understood. This problem was observed only in the presence of collisions and is known to scale with instantaneous luminosity. To reject the noise bursts an offline time window veto procedure is implemented, which vetoes events in a window of $250 \mathrm{~ms}$ around noise bursts. The duration of this window was chosen conservatively on purpose, as the data loss is limited in any way. But there is some room for improvement if the noise bursts yield increases with luminosity.
303

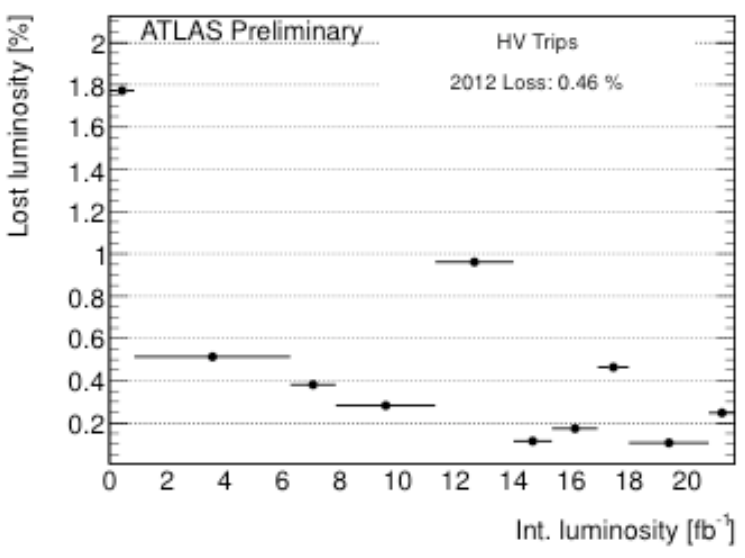

Fig. 6. Evolution of the luminosity loss associated to HV trips during 2012 data taking. During the month of September 2012 an hardware problem occurred in a module feeding a sector of the ElectroMagnetic End Cap PreSampler (EMECPS) in the $\mathrm{C}$ side of Atlas, leading to an increase of the overall trip rate.

\begin{tabular}{c|c|c}
\hline & $\mathbf{2 0 1 1}$ & $\mathbf{2 0 1 2}$ \\
\hline Total n. of trips & 355 & $\begin{array}{c}176+50 \text { Current Control } \\
\text { events }\end{array}$ \\
\hline Trip rate & $\begin{array}{c}0.27 \text { trips/Stable Beam } \\
\text { hour }\end{array}$ & $\begin{array}{c}0.103 \text { trips/Stable Beam } \\
\text { hour }\end{array}$ \\
\hline $\begin{array}{c}\text { Data rejection due to HV } \\
\text { trips }\end{array}$ & $0.96 \%$ & $0.46 \%$ \\
\hline Overall Data Quality loss & $3.2 \%$ & $0.88 \%$ \\
\hline
\end{tabular}

Table1. Comparison of the data quality performances concerning the HV trips and the overall data quality loss in two different years of ATLAS run, 2011 and 2012.

\section{CONCLUSIONS}

The LAr calorimeter system has performed at very high efficiency as well as excellent performance and stability over the first three years of operation, contributing to the overall ATLAS performance that culminated in the discovery in 2012 of Higgs boson. This performance is the result of an important operation and monitoring effort. The development and the installation of a novel HV power supply modules has significantly improved the data loss due to the HV trips, reaching the $0.46 \%$ in the last year of operation (2012). Studies are continuing in order to improve the performance as the LHC delivers data at unprecedented energies and luminosity, ensuring the full discovery potential of this instruments in the coming years.

\section{REFERENCES}

[1] The ATLAS Collaboration, The ATLAS Experiment at the CERN Large Hadron Collider, JINST 3 (2008) S08003.

[2] The ATLAS Collaboration, Liquid Argon Calorimeter Technical Design Report, CERN/LHCC 96-41 (1996).

[3] The ATLAS Collaboration, Readiness of the ATLAS Liquid Argon Calorimeter for LHC Collisions, Eur. Phys. J. C 70 (2010) 723-753. 
333 [4] W. Bonivento, Online luminosity monitoring with liquid Argon

334

335

336

337

338

339

340

341

342 calorimeters at ATLAS and D0. ATL-LARG-2001-001, (2000).

[5] S. Arfaoui, L. Hervas, and E. Monnier, The ATLAS liquid argon calorimeter high-voltage system: commissioning, optimisation, and LHC relative luminosity measurement. oai:cds.cern.ch:1394247. PhD thesis, Aix-Marseille III U., Geneva, (2011).

[6] The ATLAS Collaboration, Luminosity Determination in $p p$ Collisions at $\sqrt{ }=7 \mathrm{TeV}$ using the ATLAS detector in 2011, ATLASCONF-2011-116, (2011). 